Check for updates

Cite this: Mater. Adv., 2021,

2, 948

Received 9th November 2020,

Accepted 13th January 2021

DOI: $10.1039 / \mathrm{d} 0 \mathrm{ma} 00874 \mathrm{e}$

rsc.li/materials-advances

\section{Reduced graphene oxide integrated poly(ionic liquid) functionalized nano-fibrillated cellulose composite paper with improved toughness, ductility and hydrophobicity $\dagger$}

\author{
Rama K. Layek, (D) *ab Vijay Singh Parihar, (D) ${ }^{c}$ Jukka Seppälä, ${ }^{d}$ Alexander Efimov, (D) e \\ Sarianna Palola, (D) a Mikko Kanerva, ${ }^{a}$ Shambhavee Annurakshita, (D) ${ }^{f}$ \\ Minna Kellomäki iD ${ }^{c}$ and Essi Sarlin ${ }^{a}$
}

Reduced graphene oxide (rGO) integrated nano-fibrillated cellulose (NFC) grafted poly(ionic liquid) (PIL) composite paper with enhanced hydrophobicity, ductility and toughness was fabricated by vacuum filtration of an aqueous dispersion of rGO/NFC-graft-PIL (NFC-g-PIL). The NFC-g-PIL was synthesized by growing PIL chains from the NFC surface using free radical polymerization of a pyridinium acrylatebased ionic liquid monomer.

Nano-fibrillated cellulose (NFC) is an extremely interesting material class due to its environmental greenness, renewable resources, abundant feedstock, lightweight, high aspect ratio, optical transparency, and excellent strength and stiffness., NFC has several oxygen-containing functional groups on the surface that can bind the nanofibrils together via $\mathrm{H}$-bonding interaction to produce strong paper-like structures ${ }^{2,3}$ that are very promising for the replacement of the fossil fuel-derived synthetic plastics in the near future. Recently, NFC based composite papers have been attractive to replace many petroleum-derived polymers for various demanding applications ${ }^{3,4}$ due to their excellent properties.

\footnotetext{
${ }^{a}$ Tampere University, Engineering Material Science, P.O. Box 589, 33101 Tampere, Finland. E-mail: rama.layek@tuni.fi; Tel: +358504478355

${ }^{b}$ Department of Separation Science, School of Engineering Science,

LUT University, Mukkulankatu 19, 15210 Lahti, Finland.

E-mail: rama.layek@lut.fi

${ }^{c}$ Biomaterials and Tissue Engineering Group, BioMediTech, Faculty of Medicine and Health Technology, Tampere University, 33720 Tampere, Finland

${ }^{d}$ Polymer Technology, School of Chemical Engineering, Aalto University, Finland

${ }^{e}$ Faculty of Engineering and Natural Science, Tampere University, 33720 Tampere, Finland

${ }^{f}$ Photonics Laboratory, Tampere University, 33720 Tampere, Finland

$\dagger$ Electronic supplementary information (ESI) available: Details of the synthesis procedure and ${ }^{1} \mathrm{H}$ and ${ }^{13} \mathrm{C}$ NMR analysis of pyridinium IL, pyridinium IL acrylate, and NFC- $g$-PIL; fabrication of NFC, NFC-g-PIL, rGO1/NFC and rGO1/NFC- $g$-PIL composite papers and their digital photographs, contact angles, ${ }^{1} \mathrm{H}$ and ${ }^{13} \mathrm{C}$ NMR spectra of pyridinium IL and pyridinium IL acrylate, solid state ${ }^{1} \mathrm{H}$ and CP/MAS ${ }^{13} \mathrm{C}$-NMR spectra of NFC and NFC-g-PIL, AFM images and height profiles of NFC and NFC-g-PIL, and cross-sectional FESEM image of rGO1/NFC composite paper. See DOI: $10.1039 /$ doma00874e
}

Graphene, a multifunctional nanomaterial with a 2-D nanostructure has outstanding physical, and mechanical properties. ${ }^{5,6}$ Hence, NFC composites with graphene are gaining significant interest for various demanding applications, such as structural films, flexible electronic device packaging films, and other hybrid materials due to their promising mechanical, gas barrier and electrical properties. ${ }^{7,8}$ In this regard, a few studies are focusing on the design of graphene/NFC composites to bring new functionalities such as higher stiffness and mechanical strength, improved thermal stability and hydrophobicity, good electrical and thermal conductivity, and gas barrier properties to boost their applicability in demanding areas to replace the petroleum-derived advanced polymer composites. ${ }^{7-9}$ Graphene oxide (GO)/NFC composite papers show a remarkable improvement of mechanical properties ${ }^{10}$ due to the interfacial interaction between the hydrophilic oxygencontaining functional groups of GO and NFC. These hydrophilic oxygen-containing functional groups may absorb moisture from the environment, ${ }^{10}$ which reduces the performance of graphene/NFC composite films. The in situ reduced graphene oxide (rGO)/NFC composites and graphene nanoplates/NFC composites obtained by a vacuum-assisted assembly process display enhancement of multifunctionality ${ }^{9}$ with a decreased ductility due to nano integration and decrease of molecular mobility of the NFC chain. Hence, the majority of the graphene/nanocellulose composite films are either hydrophilic in nature, or less ductile due to the formation of a stiff and brittle composite. These are very serious issues and must be overcome before full utilization of the outstanding functionalities of graphene/NFC composite papers.

These issues of the graphene/NFC composites can be overcome by generating additional functionalities such as hydrophobicity, ductility and toughness in graphene/NFC composite films via tailoring the chemical functionalities and interfacial interaction between NFC and graphene or derivatives of graphene. It has been already reported that an ionic liquid (IL) containing hydrophobic moiety anchored on the surface of the NFC fibrils can enhance the hydrophobicity of the cellulosic derivatives. ${ }^{11}$ Besides this, IL also enhances the processability, 
physical properties, mechanical properties and ductility of the cellulosic derivatives by reducing the interactions between the cellulose fibrils. ${ }^{11}$ Recently, the scope of IL-based polymers is gaining significant attention over ILs due to their ability to improve the processability and mechanical properties within the polymeric structure over the IL monomer. ${ }^{12,13}$ Polyionic liquid (PIL) contains IL moieties in each repeating unit as it is synthesized by polymerizing the IL monomers, and can introduce new functionalities via tailoring the chemical functionalities of the PIL. Though there are numerous procedures reported to tailor the multifunctionalities of graphene/NFC composites, to the best of our knowledge, there is no report of simultaneous tailoring of the ductility, toughness, and hydrophobicity. In this communication, for the first time, we are reporting the synthesis of a novel NFC-graft-PIL (NFC- $g$-PIL) containing hydrophobic pyridyl hexyl chains and its freestanding composite paper with hydrophobic rGO. The novelty of the preparation rGO/NFC-g-PIL paper is in its ductility and hydrophobicity. The ductility of the rGO/NFC- $g$-PIL paper is related to elongation at break. The obtained rGO/NFC- $g$-PIL paper shows higher hydrophobicity and ductility, along with significant enhancement of mechanical strength with respect to NFC paper.

NFC- $g$-PIL was synthesized by growing PIL from NFC (Scheme 1) by free radical polymerization of 1-hexyl-4-(methacryloyloxy-methyl) pyridinium bromide ionic liquid (IL acrylate) using benzoyl peroxide (BP) as an initiator. ${ }^{14} \mathrm{GO}$ was synthesized by Hummers' method $^{15 a}$ and $\mathrm{rGO}$ was prepared by reducing $\mathrm{GO}$ with hydrazine hydrate in a 9:1 DMF : $\mathrm{H}_{2} \mathrm{O}$ dispersion. ${ }^{15 b}$ The free-standing $\mathrm{rGO} / \mathrm{NFC}$ and $\mathrm{rGO} /$ NFC- $g$-PIL composite papers were prepared by a vacuum-assisted assembly procedure (details in the ESI, $\dagger$ Experimental section). The required amount of rGO dispersion (1 wt \% with respect NFC) was mixed with $500 \mathrm{~mL}$ of aqueous dispersion of NFC $\left(1.4 \mathrm{mg} \mathrm{mL}^{-1}\right)$ and NFC-g-PIL $\left(1.4 \mathrm{mg} \mathrm{mL}{ }^{-1}\right)$ and sonicated for 30 minutes to obtain uniform rGO/NFC and rGO/NFC-g-PIL solutions. Finally, the

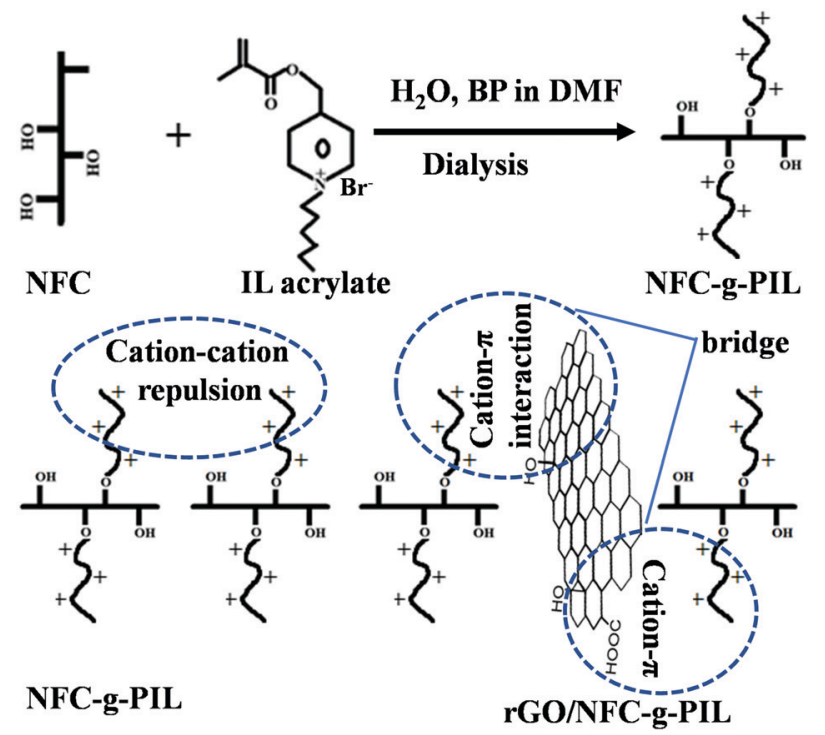

Scheme 1 Synthesis of NFC-g-PIL and interaction of rGO with NFC-g-PIL.
rGO/NFC and rGO/NFC- $g$-PIL composite films were obtained by vacuum filtration followed by compression at $70{ }^{\circ} \mathrm{C}$ and named rGO1/NFC and rGO1/NFC-g-PIL (number 1 represent wt $\%$ of rGO w.r.t. NFC and NFC- $g$-PIL). Pure NFC and NFC- $g$-PIL films were also obtained from the $1.4 \mathrm{mg} \mathrm{mL}^{-1}$ aqueous NFC and NFC- $g$-PIL dispersions, respectively, without using any rGO for comparison. The detailed synthesis procedure of NFC- $g$-PIL and fabrication of NFC, NFC- $g$-PIL paper, rGO1/NFC and rGO1/NFC- $g$-PIL composite papers and their digital photographs (ESI, $\dagger$ Fig. S1) are presented in the ESI. $\dagger$

The ${ }^{1} \mathrm{H}$ and ${ }^{13} \mathrm{C}$ NMR spectra of pyridinium IL and pyridinium IL acrylate are presented in the ESI, $\dagger$ Fig. $\mathrm{S} 2-\mathrm{S} 5$ respectively. The detail interpretation of NMR $\left({ }^{1} \mathrm{H}\right.$ and $\left.{ }^{13} \mathrm{C}\right)$ spectra is presented in the Experimental section of the ESI, $\dagger$ and it confirms the synthesis of pyridinium IL and pyridinium IL acrylate. The grafting of PIL from the NFC surface was confirmed by comparing solid state ${ }^{1} \mathrm{H}-\mathrm{NMR}$ spectra of pure NFC with NFC- $g$-PIL, where the distinguished aromatic pyridine ring protons and aliphatic hexyl protons of the repeating unit of pyridinium IL peaks clearly appear in the spectra (ESI, $\dagger$ Fig. S6 and S8). The grafting of PIL was further supported by comparing solid-state cross-polarization magic angle spinning Carbon-13 NMR (CP/MAS ${ }^{13} \mathrm{C}-\mathrm{NMR}$ ) of pure NFC with NFC- $g$-PIL in the ESI, $\dagger$ Fig. S7 and S9.

NFC is a hydrophilic biopolymer that contains several hydroxyl groups on the surface and hence, the sonication of NFC in an aqueous medium produces a stable aqueous suspension. The vacuum filtration of these aqueous suspensions produces a free-standing NFC film through the H-bonding interaction and NFC- $g$-PIL film through the H-bonding and electrostatic interactions. The sonication of rGO dispersion with an aqueous suspension of NFC and NFC- $g$-PIL produced homogeneous aqueous suspensions of $\mathrm{rGO} / \mathrm{NFC}$ and $\mathrm{rGO} / \mathrm{NFC}$ $g$-PIL. The vacuum-assisted composite formation assembly of $\mathrm{rGO} / \mathrm{NFC}$ is governed by the H-bonding and hydrophobic interaction; whereas the composite formation assembly of rGO/NFC- $g$-PIL by H-bonding, hydrophobic and cation- $\pi$ interactions. $^{16}$

In order to investigate the interfacial interaction between rGO and NFC- $g$-PIL; the Fourier transform infrared spectroscopy (FTIR) studies of the NFC, NFC- $g$-PIL, rGO1/NFC and rGO1/NFC- $g$-PIL composite papers were carried out and the recorded spectra are shown in Fig. 1(A). The NFC exhibits a typical intense and broad peak at $3310 \mathrm{~cm}^{-1}$ for the stretching vibrations of inter and intramolecular $\mathrm{H}$-bonded $-\mathrm{OH}$ groups and the $\mathrm{C}-\mathrm{H}$ stretching vibration peak in the region of $3000-2800 \mathrm{~cm}^{-1} .{ }^{17}$ This H-bonded -OH stretching peak of NFC is shifted in NFC- $g$-PIL from $3310 \mathrm{~cm}^{-1}$ to $3325 \mathrm{~cm}^{-1}$ and becomes less broad in NFC- $g$-PIL. This indicates that interfibrillar H-bonding interaction between the hydroxyl groups is reduced in NFC- $g$-PIL due to the grafting of PIL from the NFC surface. Besides this, NFC- $g$-PIL also shows a characteristic peak of polymethyl IL acrylate at $1738 \mathrm{~cm}^{-1}$ for the $-\mathrm{C}=\mathrm{O}$ stretching vibration of ester groups. ${ }^{6}$ The $\mathrm{C}-\mathrm{H}$ stretching vibration peak in the region of $3000-2800 \mathrm{~cm}^{-1}$ in NFC-g-PIL increases due to the presence of the hexyl chain in the 

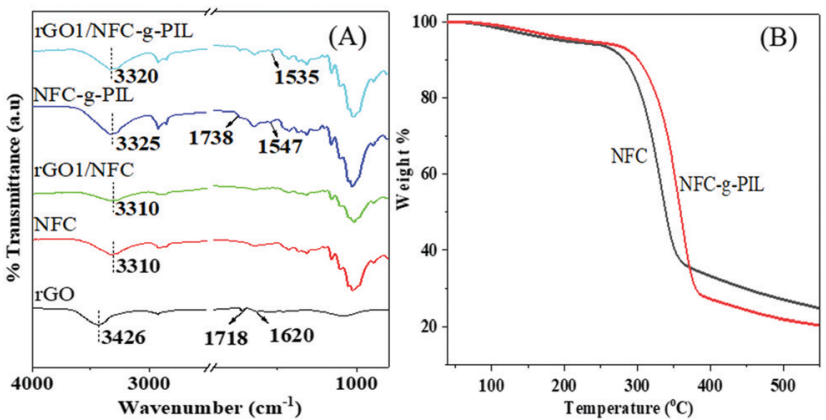

Fig. 1 (A) FTIR spectra of rGO, NFC, rGO1/NFC, NFC-g-PIL and rGO1/ NFC-g-PIL; and (B) TGA of NFC and NFC-g-PIL.

pyridinium ring and methyl group in polymethyl IL acrylate. rGO shows a characteristic peak at $\sim 1718 \mathrm{~cm}^{-1}, \sim 3426 \mathrm{~cm}^{-1}$ and $\sim 1620 \mathrm{~cm}^{-1}$ for the $\mathrm{C}=\mathrm{O}$ stretching vibration of the $-\mathrm{COOH}$ group, stretching vibration of $-\mathrm{OH}$ groups and skeletal vibration of the unoxidized graphitic domain. ${ }^{6,17}$ In the $\mathrm{rGO} /$ NFC composites the -OH stretching vibration peak appeared in the same position as for pure NFC. The - $\mathrm{OH}$ stretching vibration band in $\mathrm{rGO} / \mathrm{NFC}-\mathrm{g}$-PIL is also shifted from $3325 \mathrm{~cm}^{-1}$ to $3320 \mathrm{~cm}^{-1}$ due to the $\mathrm{H}$-bonding and ionic interaction. The NFC-g-PIL displays a typical peak at $1547 \mathrm{~cm}^{-1}$ for $\mathrm{N}^{+}-\mathrm{C}$ bond of PIL. In rGO/NFC- $g$-PIL composites, this $\mathrm{N}^{+}-\mathrm{C}$ stretching vibration band is shifted from $1547 \mathrm{~cm}^{-1}$ to $1535 \mathrm{~cm}^{-1}$ and this may be due to the cation- $\pi$ interaction between the cationic PIL of NFC-g-PIL and $\pi$-cloud of an aromatic rGO ring (Scheme 1 ). ${ }^{16}$ These results demonstrate that rGO sheets bridge interfibrillated NFC- $g$-PIL via H-bonding, ionic and cationic- $\pi$ interactions.

The TGA thermograms of NFC, and NFC-g-PIL are displayed in Fig. 1(B). The NFC shows a very small amount of weight loss below $100{ }^{\circ} \mathrm{C}$ due to the removal of associated water molecules and a sharp weight loss in the temperature range of 330 and $550{ }^{\circ} \mathrm{C}$ due to dehydration and decomposition of the NFC moiety. ${ }^{18}$ The NFC-g-PIL film shows a relatively higher weight loss compared to the pure NFC film in this temperature range. This may be due to the degradation of the PIL moiety of NFC-gPIL along with the dehydration and decomposition of the NFC moiety in the same temperature range. ${ }^{13}$

To investigate the morphology and height profile of the individual NFC and NFC-g-PIL fibers, the AFM image and the corresponding height profile of NFC and NFC-g-PIL are presented in the ESI, $\dagger$ Fig. S10(A) and (B). The AFM image and the corresponding height profile of pure NFC show that most of the NFC fibrils have a diameter of less than $10 \mathrm{~nm}$, but very few thicker NFC fibrils with aggregated bundle structures are observed. The grafting of PIL from the NFC surface does not alter the fibrillar structure of NFC; however, both the image and height profile of NFC-g-PIL show somewhat higher thickness than that of pure NFC. The increase of thickness of NFC- $g$-PIL fibers compared to NFC fibers proved that the PIL chain has been grafted from the NFC surface. The grafting is also evident from the solid state ${ }^{1} \mathrm{H}$ and ${ }^{13} \mathrm{C}$ NMR, and FTIR spectroscopy results. The AFM image (Fig. 2(A)) of rGO was taken by casting a
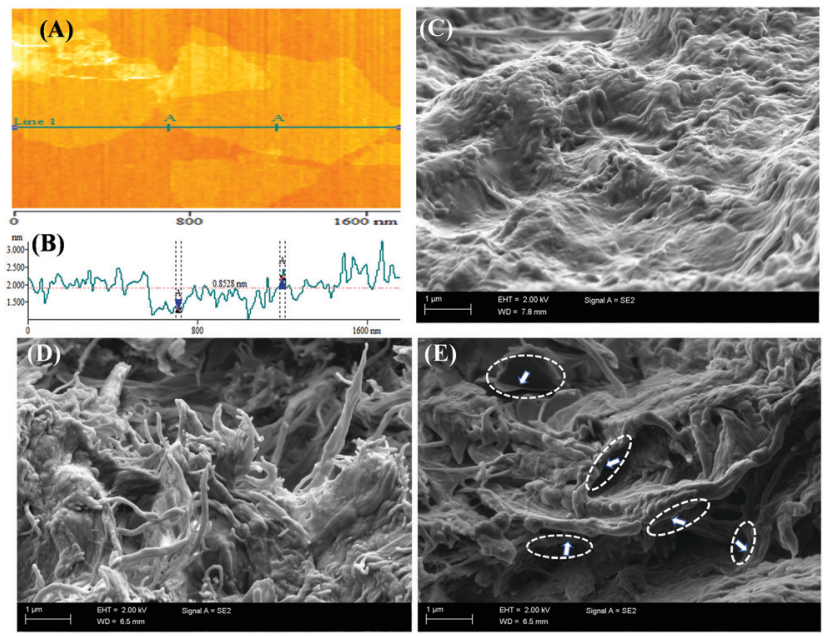

Fig. 2 (A) AFM image of $r G O$ and (B) corresponding height profile; FESEM image of the cross-section of (C) NFC; (D) NFC-g-PIL and (E) the rGO1/ NFC-g-PIL composite.

drop of dilute $\mathrm{H}_{2} \mathrm{O}: \operatorname{DMF}(9: 1)$ solution on a mica substrate. These results show a sheet-like structure of rGO; and the corresponding height profile (Fig. 2(B)) shows an rGO sheet height of $\approx 0.85 \mathrm{~nm}$, which indicates that the $\mathrm{rGO}$ sheets remain highly exfoliated in the dilute $\mathrm{H}_{2} \mathrm{O}: \operatorname{DMF}(9: 1)$ solution. ${ }^{15 b}$ In order to investigate the cross-sectional morphology and structure of the materials, scanning electron microscopy (FESEM) images of cryo fractured cross-sections of NFC, NFC- $g$-PIL, rGO1/NFC and rGO1/NFC- $g$-PIL composites are shown in Fig. S2 and the ESI, $\uparrow$ Fig. S11. Fig. 2(C) and (D) show the morphology of the assembled fiber of NFC and NFC- $g$-PIL respectively. These assembled fiber formations were observed due to the stacking of NFC and NFC- $g$-PIL fibrils together during the vacuum filtration process. From Fig. 2(E), it is noticeable that the exfoliated rGO sheets are well dispersed throughout the NFC- $g$-PIL matrix due to strong $\mathrm{H}$-bonding, electrostatic and cation- $\pi$ interactions. ${ }^{16}$ The rGO sheets also display good dispersion throughout the NFC matrix in rGO1/ NFC composites (ESI, $\dagger$ Fig. S11) due to the hydrophobic interaction. ${ }^{4}$

To investigate the hydrophobicity of the rGO1/NFC-g-PIL, the water droplet contact angle results of the NFC, NFC- $g$-PIL, rGO 1/NFC and rGO1/NFC-g-PIL are shown in Fig. 3(A) and ESI, $\dagger$ Table S1. Pure NFC shows a contact angle of $49 \pm 2^{\circ}$ indicating that it is hydrophilic in nature. Due to the grafting of PIL from the NFC surface, the water contact angle values increased from $49 \pm 2^{\circ}$ to $65 \pm 1.5^{\circ}$ for NFC- $g$-PIL. The rGO1/ NFC and rGO1/NFC- $g$-PIL composites display contact angles of $66 \pm 1^{\circ}$ and $75 \pm 1^{\circ}$. It is reported that graphene sheets have outstanding hydrophobicity ${ }^{19}$ and hence, successful integration of rGO improved the hydrophobicity of rGO1/NFC and rGO1/NFC-g-PIL composites in this study.

To examine the mechanical behaviour of the composite films, the stress-strain curves of the NFC, NFC-g-PIL, rGO1/ NFC and rGO1/NFC- $g$-PIL composites are presented in Fig. 3(B). Due to the presence of the strong H-bonding 

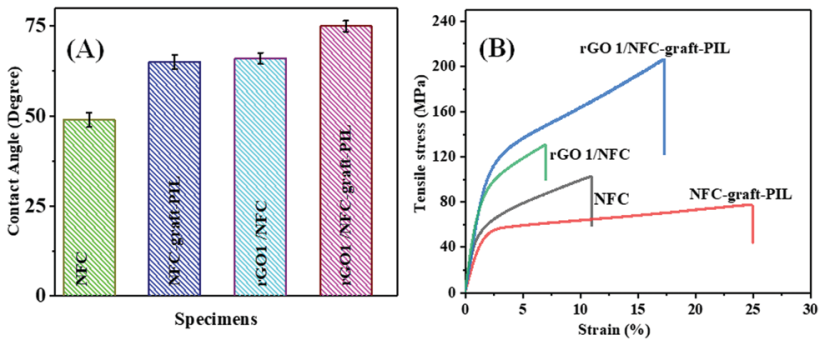

Fig. 3 (A) The contact angle and (B) the stress-strain curve of NFC, NFC-g-PIL, rGO1/NFC and rGO1/NFC- $g$-PIL composites.

interaction between NFC nanofibers, the pure NFC film shows an excellent tensile strength of $105 \pm 5 \mathrm{MPa}$, Young's modulus of $6.1 \pm 0.30 \mathrm{GPa}$, and toughness of $9.21 \mathrm{MJ} \mathrm{m} \mathrm{m}^{-3}$ with an elongation at break of $12 \pm 1.5 \%$. For the NFC- $g$-PIL film, the tensile strength decreases from $105 \pm 5 \mathrm{MPa}$ to $80 \pm 4.3 \mathrm{MPa}$ and the Young's modulus from $6.1 \pm 0.30 \mathrm{GPa}$ to $3.5 \pm 0.20 \mathrm{GPa}$. However, the elongation at break and toughness of the NFC- $g$-PIL film increase from $12 \pm 1.5 \%$ to $25 \%$ and $9.21 \mathrm{MJ} \mathrm{m}^{-3}$ to 15.9 $\mathrm{MJ} \mathrm{m^{-3 }}$ respectively. The covalently grafted cationic PIL remains anchored over the NFC surface via electrostatic interaction and possibly weakens the interfibrillar interactions due to electrostatic repulsion between the cationic PIL of the NFC- $g$-PIL compared to pure NFC causing the decrease of tensile strength and Young's modulus but with a significant enhancement of ductility and toughness. It is reported that, with the introduction of multifunctional fillers, the ductility of the NFC composite films decreased significantly and produced a very brittle composite film with respect to pure NFC., ${ }^{90}$ The rGO1/NFC composite shows a tensile strength of $135 \pm 3 \mathrm{MPa}$, Young's modulus of $11 \pm 0.40 \mathrm{GPa}$, toughness of $6.70 \mathrm{MJ} \mathrm{m}^{-3}$ and elongation at break of $6 \pm 1.5 \%$. Hence, the nano-integration of rGO into the NFC structure makes the material more brittle and decreases its ductility significantly. However, in rGO1/NFC- $g$-PIL composites, the rGO sheets interact with NFC-g-PIL chains by multiple level molecular interactions, such as cation- $\pi$ interaction and $\mathrm{H}$-bonding interaction, and bridge the NFC- $g$-PIL molecules together. This leads to the formation of a ductile composite with a significant improvement in the tensile strength (200 $\pm 4 \mathrm{MPa})$ and Young's modulus (5.2 $\pm 0.30 \mathrm{GPa})$. It is reported in the literature that toughness values of NFC and NFC based composite papers can vary from $6.62-51 \mathrm{MJ} \mathrm{m}^{-3}$ depending on several factors such as the source, and origin of the NFC fibers, processing of the NFC fibers and methods of fabrication of the NFC and NFC based composite film. ${ }^{21}$ Our pure NFC paper shows a toughness of 9.21 $\mathrm{MJ} \mathrm{m}^{-3}$, whereas the obtained NFC- $g$-PIL film and rGO/NFC- $g$-PIL film show a considerable toughness of $15.9 \mathrm{MJ} \mathrm{m}^{-3}$ and $25.3 \mathrm{MJ} \mathrm{m}^{-3}$ respectively with a significant enhancement of elongation at break of 50\% and 108\% compared to pure NFC films. It also signifies that our NFC- $g$-PIL film and rGO/NFC- $g$-PIL film have very good ductility. The interfibril connection of NFC-g-PIL with rGO by noncovalent cation $-\pi$ interaction and H-bonding interaction may offer a chance of sliding the NFC- $g$-PIL over the rGO surface during mechanical elongation and this may lead to the significant enhancement of mechanical properties of rGO/NFC- $g$-PIL compared to pure NFC and NFC-g-PIL film.
In conclusion, we have successfully synthesized NFC- $g$-PIL by simple free radical polymerization of a pyridinium acrylatebased IL monomer from the NFC surface. This NFC-g-PIL shows good dispersion in an aqueous medium. For the first time, we used this NFC- $g$-PIL for the fabrication of rGO/NFC- $g$-PIL composite paper by a vacuum-assisted assembly procedure. rGO acts as a bridge in binding the NFC- $g$-PIL fibrils by cation- $\pi$ interaction and it governs the fabrication of rGO1/NFC- $g$-PIL composite paper. Our free-standing, rGO/NFC-g-PIL composite paper exhibits a substantial improvement of mechanical strength, toughness, and ductility along with hydrophobicity compared to pure NFC film. Therefore, this highly flexible rGO integrated NFC- $g$-PIL composite film may provide a new direction for the development of multifunctional hydrophobic NFC composites with enhanced sustainability and boost the future of biocomposite based bioeconomy.

\section{Conflicts of interest}

There are no conflicts to declare.

\section{Acknowledgements}

This work was supported by the Academy of Finland prostdoctoral project (project no-326453) and Center of Excellence in Body-OnChip Research (project no. 312409).

\section{References}

1 D. Trache, M. H. Hussin, M. K. Mohamad Haafiz and V. K. Thakur, Nanoscale, 2017, 9, 1763.

2 N. Lavoine and L. Bergstrom, J. Mater. Chem. A, 2017, 5, 16105.

3 M. Henriksson, L. A. Berglund, P. Isaksson, T. Lindstrom and T. Nishio, Biomacromolecules, 2008, 9, 1579.

4 J. Malho, P. Laaksonen, A. Walther, O. Ikkala and M. B. Linder, Biomacromolecules, 2012, 13, 1093.

5 R. K. Layek, K. R. Ramakrishnan, E. Sarlin, O. Orell, M. Kanerva, J. Vuorinen and M. Honkanen, J. Mater. Chem. A, 2018, 6, 13203.

6 R. K. Layek and A. K. Nandi, Polymer, 2013, 54, 5087.

7 Q. Jiang, C. Kacica, T. Soundappan, K. Liu, S. Tadepalli and P. B. S. Singamaneni, J. Mater. Chem. A, 2017, 5, 13976.

8 J. Duan, S. Gong, Y. Gao, X. Xie, L. Jiang and Q. Cheng, ACS Appl. Mater. Interfaces, 2016, 8, 10545.

9 L. N. Dang and J. Seppala, Cellulose, 2015, 22, 1799.

10 A. Kafy, A. Akther, M. I. R. Shishir, H. C. Kim, Y. Yun and J. Kim, Sens. Actuators, A, 2016, 247, 221.

11 M. Shimizu, T. Saito, H. Fukuzumi and A. Isogai, Biomacromolecules, 2014, 15, 4320.

12 J. Yuan and M. Antonietti, Polymer, 2011, 52, 1469.

13 K. Grygiel, B. Wicklein, Q. Zhao, M. Eder, T. Pettersson, L. Bergstrom, M. Antoniettia and J. Yuan, Chem. Commun., 2014, 50, 12486.

14 D. Roy, M. Semsarilar, J. T. Guthriea and S. Perrier, Chem. Soc. Rev., 2009, 38, 2046. 
15 (a) W. S. Hummers, Jr. and R. E. Offeman, J. Am. Chem. Soc., 1958, 80, 1339; (b) S. Park, J. An, I. Jung, R. D. Piner, S. J. An, X. Li, A. Velamakanni and R. S. Ruoff, ACS Nano, 2010, 4, 3845 .

16 H. Gao, S. Zhang, F. Lu, H. Jia and L. Zheng, Colloid Polym. Sci., 2012, 290, 1785.

17 R. K. Layek, Md. E. Uddin, N. H. Kim, A. K. T. Lau and J. H. Lee, Composites, Part B, 2017, 128, 155.

18 D. Zheng, Y. Zhang, Y. Guo and J. Yue, Polymers, 2019, 11, 1130.

19 Y. Bai, AIP Conf. Proc., 2017, 1794, 02008.

20 Z. Shen and J. Feng, ACS Appl. Mater. Interfaces, 2018, 10, 24193.
21 (a) P. Dhar, J. Phiri, G. R. Szilvay, A. Westerholm-Parvinen, T. Maloneya and P. Laaksonen, J. Mater. Chem. A, 2020, 8, 656; (b) H. Dua, M. Parita, M. Wub, X. Cheb, Y. Wang, M. Zhang, R. Wang, X. Zhang, Z. Jiang and B. Li, J. Hazard. Mater., 2020, 400, 123106; (c) M. Henriksson, L. A. Berglund, P. Isaksson, T. Lindstrom and T. Nishino, Biomacromolecules, 2008, 9, 1579; (d) Y. Zhou, C. Chen, S. Zhu, C. Sui, C. Wang, Y. Kuang, U. Ray, D. Liu, A. Brozena, U. H. Leiste, N. Quispe, H. Guo, A. Vellore, H. A. Bruck, A. Martini, B. Foster, J. Lou, T. Li and L. Hu, Mater. Today, 2019, 30, 17; (e) F. Chen, W. Xiang, D. Sawada, L. Bai, M. Hummel, H. Sixta and T. Budtova, ACS Nano, 2020, 14, 11150. 